Please do not remove this page

RMIT

UNIVERSITY

\title{
Development and flight test of an avionics LIDAR for helicopter and UAV low-level flight
}

Sabatini, Roberto; Richardson, Mark; Roviaro, Ermanno

https://researchrepository.rmit.edu.au/esploro/outputs/9921859672801341/filesAndLinks?institution=61RMIT_INST\&index=null

Sabatini, R., Richardson, M., \& Roviaro, E. (2013). Development and flight test of an avionics LIDAR for helicopter and UAV low-level flight. Journal of Aeronautics and Aerospace Engineering, 2(3), 1-13.

https://doi.org/10.4172/2168-9792.1000114

Document Version: Published Version

Published Version: https://doi.org/10.4172/2168-9792.1000114

Repository homepage: https://researchrepository.rmit.edu.au

(C) 2013 Sabatini R, et al

Downloaded On 2023/04/26 23:07:50 +1000

Please do not remove this page 
Thank you for downloading this document from the RMIT Research Repository.

The RMIT Research Repository is an open access database showcasing the research outputs of RMIT University researchers.

RMIT Research Repository: http://researchbank.rmit.edu.au/

\section{Citation:}

Sabatini, R, Richardson, M and Roviaro, E 2013, 'Development and flight test of an avionics LIDAR for helicopter and UAV low-level flight', Journal of Aeronautics and Aerospace Engineering, vol. 2, no. 3, pp. 1-13.

See this record in the RMIT Research Repository at:

https://researchbank.rmit.edu.au/view/rmit:22672

Version: Published Version

\section{Copyright Statement:}

(C) 2013 Sabatini $R$, et al

Link to Published Version:

http://dx.doi.org/10.4172/2168-9792.1000114 


\title{
Development and Flight Test of an Avionics Lidar for Helicopter and UAV Low-Level Flight
}

\author{
Roberto Sabatini ${ }^{1 *}$, Mark A Richardson ${ }^{2}$ and Ermanno Roviaro ${ }^{3}$ \\ ${ }^{1}$ Department of Aerospace Engineering, Cranfield University, Cranfield, Bedford MK43 OAL, UK \\ ${ }^{2}$ Defence Academy of the UK, Cranfield University, Shrivenham, Swindon SN6 8LA, UK \\ ${ }^{3}$ Electro-Optics R\&D Laboratories, SELEX-ELSAG, Cogoleto (Genova) 16016, Italy
}

\begin{abstract}
In recent years, laser radar (LIDAR) has become a promising technology for navigation and obstacle avoidance in helicopters and UAV, mainly because of its good wire detection performance on a wide range of incidence angles, and also due to its outstanding range and accuracy. In this paper we describe the activities carried out for the design, integration and test of the Laser Obstacle Avoidance System "Marconi" (LOAM) on helicopter and UAV platforms. After a brief description of the system architecture and sensor characteristics, emphasis is given to the performance models and processing algorithms required for obstacle detection/classification and calculation of alternative flight paths, as well as to the ground and flight test activities performed on various platforms.
\end{abstract}

Keywords: LIDAR; Laser obstacle detection; Laser warning system; UAV low-level flight; Helicopter low-level flight

\section{Introduction}

In order to achieve mission effectiveness in the present threat environment, military helicopters and small Unmanned Aerial Vehicles (UAVs) operations are focusing on low-level or nap-of-the-earth flying. This is the tactic of employing the aircraft in such a manner as to utilize the terrain profile to enhance survivability by degrading the enemy's ability to visually, optically or electronically detect or locate the aircraft. In these scenarios, radar is normally required to maintain the aircraft flight at a present altitude above the terrain. Since the adoption of this philosophy, the incidence of obstacle strike accidents has grown. The main restrictions for low-level navigation and terrain following operations with helicopters and UAV are due to adverse weather conditions. Low visibility is the main reason that prevents flight/ground crews from safely controlling the aircraft and from identifying possible obstacle collision hazards. The first laser experiment directed towards a laser obstacle detection and avoidance system started in 1965 with a Nd:YAG laser [1]. This system demonstrated the feasibility of using lasers to detect obstacles such as wires. Semiconductor lasers, such as GaAs and GaAlAs have been experimented with since 1966 [2]. These lasers radiate in the wavelength region of 0.84 to $0.9 \mu \mathrm{m}$. The experience gained with these experimental systems pointed out many features that are now being incorporated into present day research. Due to eye-safety and adverse weather (fog) propagation concerns, further development with Nd:YAG and the various semiconductor lasers has been substantially reduced, in favour of $\mathrm{CO}_{2}$ lasers. One of the first heterodyne detection $\mathrm{CO}_{2}$ system was the LOWTAS, developed by UTRC. More recent developments include CLARA, the Anglo-French compact laser radar demonstrator program [3]; HIWA, a German system built and tested by Eltro and Dornier [4]; and OASYS, developed in the U.S. by Northrop [5]. Current research efforts are concentrating on $1.54 \mu \mathrm{m}$ (Raman-shifted Nd:YAG and Er:glass) solid state lasers. One $1.54 \mu \mathrm{m}$ system is being developed for the Italian Military Forces by Marconi S.p.A., in cooperation with the Air Force Flight Test Centre. The equipment, here named LOAM (Laser Obstacle Avoidance System "Marconi"), is a low-weight/volume navigation aid system for rotary-wing/UAV platform specifically designed to detect potentially dangerous obstacle placed in or nearby the flight trajectory and to warn the crew in suitable time to implement effective avoiding manoeuvres.
The first airborne prototype of the LOAM has been assembled and extensive laboratory and field tests have been performed on the various sub-units, in order to refine the system design (both hardware and software components). Furthermore, the overall system is now being tested in flight.

\section{Operational Requirements for an OWS}

For an Obstacle Warning System (OWS) to be effective it must meet certain requirements. The first and most important requirement is reliable detection of all obstacles at almost all angles of incidence of radiation with a very high probability of detection and very low false alarm rate. By all obstacles, it is meant terrain masses, buildings, poles, towers, power cables and indeed any structure which may pose a hazard to low/fast flying aircraft.

The need for a high probability of detection is obvious since no obstacle must go undetected. A low false alarm rate is required to prevent spurious warnings that would cause the pilot to increase his altitude without real need, thus making him a better target.

Another operational requirement is the minimum detection range. This will depend upon the aircraft speed, climb angle capability (different for helicopter, UAV and airplane platforms), and pilot reaction time. As an example, for an airplane flying straight and level at $300 \mathrm{~m} / \mathrm{sec}$ and allowing a reasonable pilot reaction time and aircraft response time of between five to ten seconds, detection ranges of about two to three kilometres are adequate. For helicopter and small UAV applications, this range is generally reduced by an order of magnitude or more.

*Corresponding author: Roberto Sabatini, Department of Aerospace Engineering, Cranfield University, Cranfield, Bedford MK43 OAL, UK, E-mail: r.sabatini@cranfield.ac.uk

Received May 01, 2013; Accepted May 23, 2013; Published May 31, 2013

Citation: Sabatini R, Richardson MA, Roviaro E (2013) Development and Flight Test of an Avionics Lidar for Helicopter and UAV Low-Level Flight. J Aeronaut Aerospace Eng 2: 114. doi:10.4172/2168-9792.1000114

Copyright: (c 2013 Sabatini R, et al. This is an open-access article distributed under the terms of the Creative Commons Attribution License, which permits unrestricted use, distribution, and reproduction in any medium, provided the original author and source are credited. 
The system should, ideally, perform all of its required functions in all weather, day and night. In practice however, laser radiation is not capable of all-weather operation and the best trade-off of system characteristics must be looked at.

\section{LOAM General Description}

The LOAM system is capable of detecting obstacles placed in or nearby the helicopter/UAV trajectory, classifying/prioritising the detected obstacles, and providing obstacle warnings and information to the crew (both aural and visual). The system laser beam scans periodically the area around the flight trajectory inside a FOV of $40^{\circ}$ in azimuth and $30^{\circ}$ in elevation with field of regard capability of $\pm 20^{\circ}$ both on azimuth and elevation, centred on the optical axis of the system (Figure 1).

Furthermore, LOAM allows the operator to select the azimuth orientation of the FOV among three possible different directions (Figure 2).

This allows for the optical axis to be oriented either in the same direction of the platform "heading" (normal flight envelope), or $20^{\circ}$

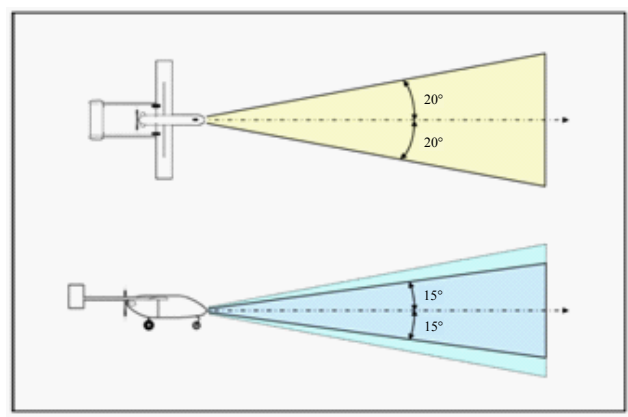

Figure 1: LOAM Horizontal and Vertical FOV.

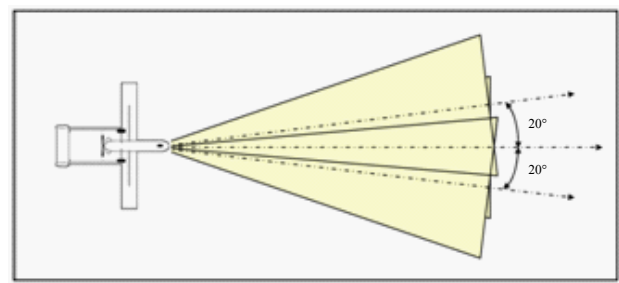

Figure 2: LOAM FOV Orientation.

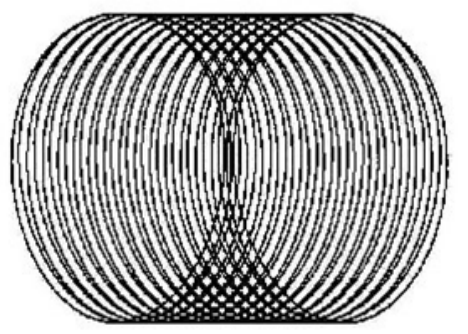

Figure 3: LOAM Scan Pattern. left/right with respect to the platform "heading" (to optimise coverage during turning manoeuvres at high angular speed). During every scan ( $4 \mathrm{~Hz}$ repetition frequency), the laser beam changes its orientation producing an elliptical pattern across the FOV with the characteristics shown in Figure 3.

After various experiments performed with different patterns, the scanned elliptical pattern was selected. The main advantages of this scanning pattern are:

- It is well adapted to the detection of most dangerous obstacles, like wires, due to the several and evenly qually spaced vertical lines

- It can be realised with a very reliable scan mechanics with reduced weight.

Using dedicated signal processing algorithms optimised for lowlevel obstacle detection, the system holds an inherently high capacity to detected various types of obstacle independently from the platform motion during the frame acquisition period, providing the possibility of reconstructing the obstacle shape without using navigation data (stand-alone integration) in slow-moving platforms with a benign attitude envelope. Additionally, LOAM can be integrated with the aircraft navigation sensors if required, especially in platforms with high dynamics envelopes [6,7].

LOAM performs echo detection through an analogue process comprising an optical-electrical conversion, a signal pre-amplification and a threshold comparison. The signal pre-amplification is achieved by an automatic controlled gain amplifier to increase the system sensitivity as the elapsed time from the laser emission increases in order to adjust the sensitivity on the basis of the expected return signal power in connection with the obstacle range. Furthermore, an adjustable threshold level is provided to take into account the background conditions. These features reduce the probability of false echo detection due to the atmospheric back-scatter near the laser beam output and optimise the system sensitivity in various operational weather conditions.

LOAM performs echo analysis in order to determine the presence of possible obstacles and to determine their geometrical characteristics and position. For this purpose, LOAM operates through two sequential analysis process: local analysis and global analysis.

The "local analysis" process is performed on the single echoes in order to determine range, angular coordinates and characteristics of the obstacle portion generating them. The "global analysis" process manages groups of echoes, detected during a scan period, with the related information provided by the "local analysis" process, in order to perform the obstacle detection as a whole and determine the related shape and type. LOAM is capable to automatically classify obstacles according to the following classes [6]:

- Wire: This class groups all thin obstacles like wires and cables (e.g., telephone cables, electrical cables and cableway);

- Tree: This class groups vertical obstacles of reduced dimensions (e.g., trees, poles and pylons);

- Structure: This class groups extended obstacles (e.g., bridges, buildings and hills).

Furthermore, LOAM performs automatic prioritisation of the detected obstacles in function of the risk represented according to the relevant range, and provides the crew with timely warnings and information of the detected obstacles in order to allow the 


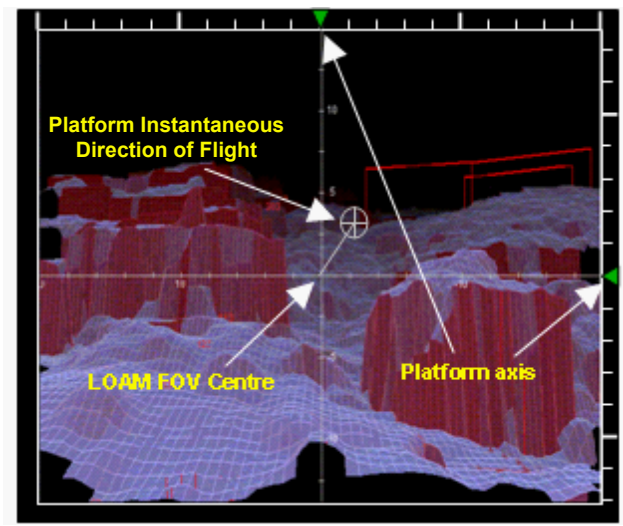

Figure 4: LOAM 3-D Display Format.

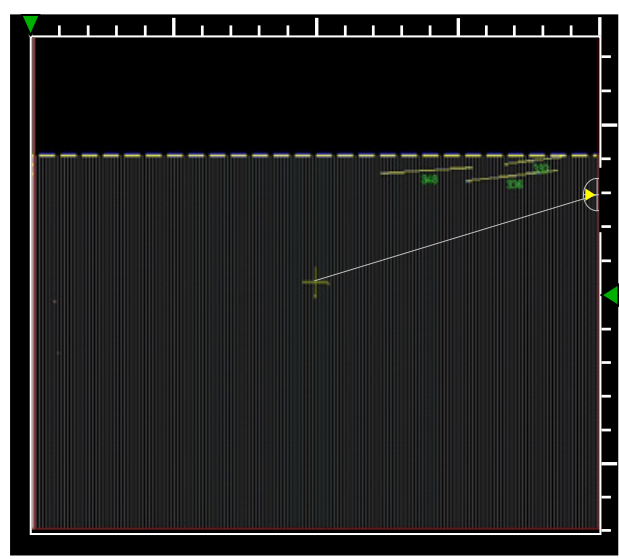

(a)

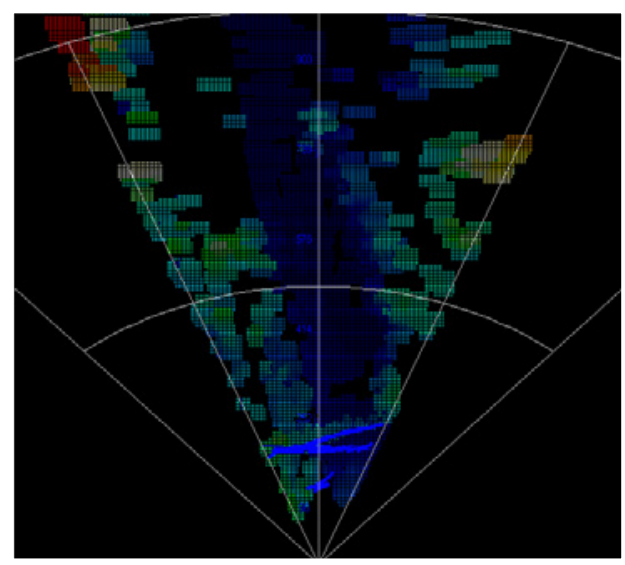

(b)

Figure 5: LOAM 2-D and Altimetric Display Format.

implementation of effective avoidance manoeuvres. For this purpose, LOAM system is able to deliver both visual and audio warnings.

LOAM information relative to the detected obstacles can be provided on a dedicated display (NVG compatible), whose screen represents the FOV of the system. Both 3-D and a 2-D are possible,

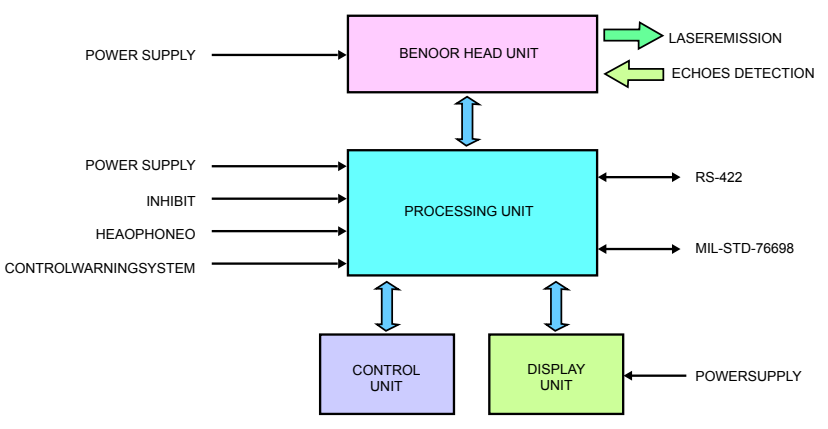

Figure 6: LOAM Architecture. Adapted from [6].

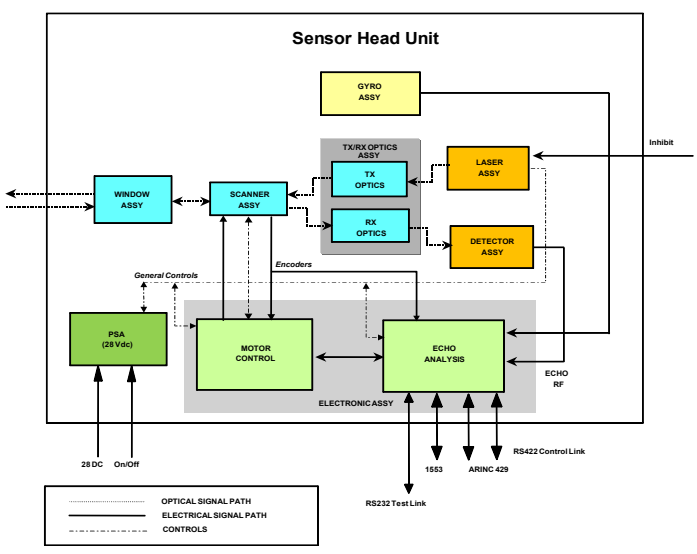

Figure 7: SHU Architecture. Adapted from [7].

together with an altimetric profile format. An example of a 3-D LOAM display format is shown in Figure 4.

The LOAM 2-D and altimetric display formats are shown in Figure $5 \mathrm{a}$ and $5 \mathrm{~b}$ respectively The general architecture of LOAM system is shown in Figure 6. LOAM main components are the Sensor Head Unit (SHU), the Control Panel (CP) and the Display Unit (DU). In the following paragraph a brief description of LOAM SHU is given, together with an outline of the main EPU functions.

\section{LOAM Sensor Head Unit Architecture}

The SHU performs the following main functions [7]:

- To generate a laser beam and scan the area around the flight trajectory;

- To detect return echoes;

- To analyse detected echoes in order to compute range, coordinates and local geometrical characteristics (attributes) of the obstacles they come from;

- To communicate echo range, coordinates and attributes to LOAM Processing Unit, or to other on board systems, via a RS-422 high speed serial data link.

The architecture of the SHU is shown in Figure 7.

As illustrated above, the SHU scans a laser beam in the area around the flight trajectory, performs echo detection through an analogue process comprising an optical-electrical conversion (by means of an avalanche photodiode-APD), a signal pre-amplification and a threshold 
comparison (adjustable threshold).

The SHU performs echo analysis in order to compute range, coordinates (azimuth, elevation with respect to LOAM reference frame) and local geometrical characteristics (attributes) of the obstacles they come from. Particularly, the following functions are performed:

- The echo angular coordinates are determined on the grounds of the scanner orientation;

- The echo range is calculated computing the "two-way" travelling time of the scan laser pulse;

- The geometrical characteristics of the echo are determined with a local "geometrical" analysis of nearby echoes along the scanner pattern and on the ground of the "absolute" power returned.

\section{The SHU interfaces include}

- One RS422 serial link to the Control Panel for controls and BIT activation

- One RS232 serial link for off-line test purpose

- One ARINC 429 to acquire H/C navigation data

- One MIL-1553-BUS to deliver obstacle properties and coordinates

- One discrete input signal to inhibit laser emission

- Two discrete output signal for audio warning.

The Window Assembly allows the transmission and the reception of the laser beam across the SHU chassis. The Window Assembly is realised with a slice of synthetic fused silica.

The Scanner Assembly integrates the $\mathrm{H} / \mathrm{W}$ resources necessary to scan the laser beam, and the virtual input pupil of the detector, throughout the overall FOV. It also allows Line of Sight (LOS) orientation. For this purpose, the Scanner Assembly comprises:

- A swash mirror mounted on an azimuth turret

- One brushless motor to allow the swash mirror motion

- One brushless motor to allow the azimuth turret motion

- One brushless motor to allow the tilt turret motion.

The swash mirror rotates at a constant speed around its axis reflecting the laser beam thus to draw a quasi-elliptical pattern in space. The turret periodically sweeps in azimuth the FOV. The composition of these two movements allows to produce the required quasi elliptical scan pattern previously described. Change in LOS orientation is achieved offsetting the central position of the periodical sweep of the

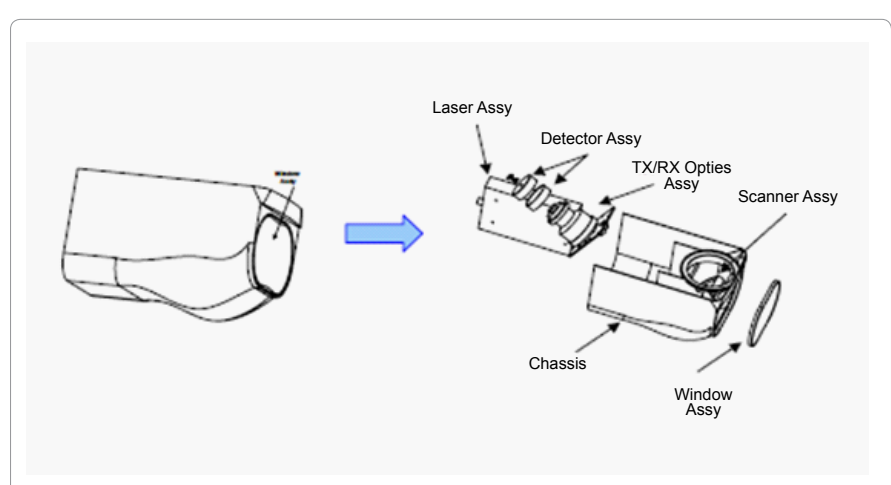

Figure 8: Sub-units arrangement inside the SHU Chassis. turret by an angular value equal to the required change.

According to the SHU architecture shown in Figure 8, the TX/RX Optics Assembly integrates the optical components necessary:

\section{In transmission}

- To collect via fiber optics the laser output power from the Laser Assembly

- To generate the scan laser beam with the required optical divergence and dimensions

- To projecting the scan laser beam on the swashing mirror of the Scanner Assembly

\section{In reception}

- To collect the echo return power reflected by the swashing mirror of the Scanner Assembly

- To focalise the collected power on the photodiode of the Detector Assembly.

To this purpose, the TX/RX Optics Assembly comprises:

\section{For transmission}

- One beam expander that provides to collect the laser output power via fiber optics and to expand and parallelise it

- One prism that allows to reflect the generated beam onto the swashing mirror with the due alignment

\section{For reception}

- One telescope that collects the echo returns power reflected by the swashing mirror of the Scanner Assembly and to focalise it on the photodiode of the Detector Assembly.

The Detector Assembly provides to detect laser echoes on the grounds of the laser return power received through the TX/RX Assembly. To this purpose, the Detector Assembly comprises an Avalanche Photodiode (APD) with related bias circuitry, a controlled gain amplifier and the threshold circuitry necessary for the echoes detection, all integrated in a single mechanical module straight connected to the telescope of the TX/RX Assembly.

The Electronic Assembly performs the following functions:

- Analyses detected echoes, received as an RF signal from the Detector Assembly

- Controls the scanner assembly motors

- Handles SHU general controls and BIT operations

- Processes the detected echoes, in order to analyse and recognise obstacle class, in order to properly alert $\mathrm{H} / \mathrm{C}$ crew

- Processes the acquired information in order to detect, isolate and calculate position and characteristics of potential obstacles

- Computes display information and symbols data

- Makes available the warning information to the Display Unit.

All the relevant electronics to accomplish the above mentioned functions is integrated in two printed circuit board.

The location of the Laser Assembly, the Detector Assembly, the TX/ RX Optics Assembly, the Scanner Assembly and the Window Assembly inside the Chassis is shown in Figure 9. The Laser Assembly provides 


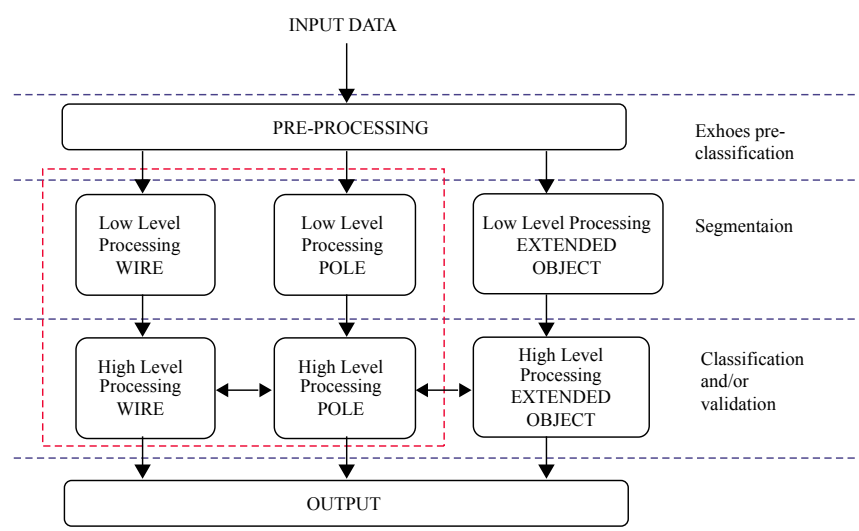

Figure 9: Algorithms Structure for Data Processing [6].

the required laser power necessary to scan operations. It comprises an Erbium doped fiber (Er:fiber) laser, the related control circuitry and power supply, all integrated in a single mechanical module. The laser power delivery to the "TX/RX Optics assembly" is provided via a fiber optics connected to the beam expander.

The Power Supply Assembly provides the power required by all SHU sub-units, except for the Laser Assembly which is directly connected to the platform mains. To this purpose, the Power Supply Assembly comprises in a single mechanical module all the circuitry necessary to interface with the platform mains and to generate output voltages as required by the SHU sub-units. The Gyro Assembly provides reference signals to the Electronic Assembly to uncouple echoes coordinates with respect to the helicopter/UAV vibration to compensate echoes angular co-ordinates, to allow a correct obstacles geometry reconstruction. The Gyro Assembly is composed by 3 gyros integrated in a single mechanical module. The Chassis is realised by a casting aluminium mechanical envelope that encloses and protect all the SHU sub-units.

In an obstacles detection and warning system, there is the need to provide only essential information to the pilot. The scanner system, in fact, detects the position of every potential obstacle in the environment where the helicopter/UAV is moving. In a generic scenario, with many obstacles in the field of view of the warning system, the pilot may have difficulties to control them. For this reason, a system capable of discriminating the most dangerous obstacles and supplying the relative information is required. To solve this problem, three algorithms have been developed for future incorporation in LOAM:

- Calculation of future trajectory

- Calculation of intersections with the obstacles

- Determination of alternative (optimal) trajectory.

Another area of development consists in the definition of efficient processing algorithms for performing Obstacle Detection and Classification Processing (ODC) at a very high speed and with high precision. In LOAM, a pre-processing is performed that, according to the range contrast between consecutive laser returns, allows a preclassification of detected obstacles. This pre-classification defines the attributes of the echoes. The subsequent processing, taking into account these attributes, performs final classification and also straightline recognition algorithms, which extract from the echoes list those related to the same structure. Particularly, two different algorithms have been developed: the first is optimised to process echoes generated by thin objects, like wires and poles, the second is optimised to process all the echoes generated by extended obstacles, like houses, trees, woods and other solid objects. These algorithms identify the boundaries of the obstacles; additional neighbourhood criteria allow distinguishing "wire-class" from "extended object" obstacle classes.

Dedicated simulation activities and actual flight tests were required to verify and refine the performance of the various processing algorithms described, as well as to determine the sensor performance in favourable and adverse weather conditions.

Some electro-optical parameters relative to the laser sub-unit are listed in Table 1.

\section{Obstacle Detection and Classification Algorithms}

As described before, LOAM anti-collision system performs obstacle detection on the basis of laser radar technique. Once the echoes energy has been optically collected, the obstacle detection is performed on the basis of an echoes analog detection and of two successive analysis processes of the detected echoes.

The first process, referred as preprocessing, is performed at a very high rate straight during the echo acquisition in order to achieve single echo specific data and to characterize it on the basis of local range contrast analysis with respect to nearby echoes. The preprocessing analyzes only echoes that are generated by obstacles that are within the fixed range not to compel the processing assembly to process unnecessary echoes.

The second process, referred as processing, is performed at a lower rate and manages groups of preprocessed echoes with the related information in order to achieve, by a two-step analysis, the final obstacle detection and the related shape and type.

Considering the current helicopter/UAV operational scenarios, wires represent by far, the most dangerous obstacle due to their low visibility to the naked eye even in good weather conditions. No present on board navigation/vision system is able to effectively aid the pilot in being warned of their presence.

In solving the problem of obstacle detection and classification for a laser radar system, the main difficulty was to find an algorithm capable to perform the processing at a very high speed and at the same time able to provide result responding to the precision requirements.

The pre-processing algorithms elaborate the range contrast between consecutive laser returns and perform a pre-classification of detected obstacles. This pre-classification defines the attributes of the echoes. The processing algorithms, taking into account these attributes performs final classification and also straight-line recognition algorithms, which extract from the echo's list, those related to the same structure.

There are two different types of processing algorithms: the first is optimized to process echoes generated by thin objects, like wires and poles, the second is optimized to process all the echoes generated by extended obstacles, like houses, trees, woods and other solid objects. These algorithms identify the boundaries of the obstacles; additional

\begin{tabular}{|c|c|c|}
\hline Parameter & Description & Value \\
\hline Wavelength & Laser emission wavelength & $1.55 \mu \mathrm{m}$ \\
\hline Peak Power & $\begin{array}{c}\text { Laser pulse power at the "Laser As- } \\
\text { sembly" output }\end{array}$ & $10 \mathrm{~kW}$ \\
\hline Pulse Duration & Laser pulse duration & 3 to $5 \mathrm{~ns}$ \\
\hline Frequency & Laser pulse repetition frequency & $60 \mathrm{kHz}$ \\
\hline
\end{tabular}

Table 1: Laser Parameters. 
neighborhood criteria allow distinguishing "wire-class" obstacles from "extended object-class" obstacles.

In order to perform its tasks the processing algorithms make use of image and data segmentation and data validation. Figure 10 shows the three levels of the processing algorithms. The processing algorithms are conceived and optimized for the quasi elliptical scanning pattern described before.

The thin object-processing algorithm works on a subset of echoes of the current frame. This algorithm processes only the echoes whose attributes, defined by pre-processing assembly, are weak echo and thin object. To process the incoming data it is not necessary to acquire the entire frame; the single echo, in fact, is processed as soon as it is acquired.

Image segmentation is the process of dividing the image into areas where the echoes are relatively uniform in range value and possible thin obstacles are created from this subset of data. Then all possible generated thin obstacles are processed so that some redundant cluster can be merged.

After the image segmentation the different clusters must be validated. This means that the detected echoes are processed by a statistical algorithm to determine if the obstacles are generated by real aligned echoes or by noising data. Also the algorithm dedicated at the detection of the extended object is divided in two different phases: echoes classification and segmentation. The echoes already classified as extended object need to be processed by a dedicated selection algorithm because many of these are not generated by a real extended obstacle (like, for example, the ground). A well-defined number of echoes, acquired in a short time range, have some geometrical characteristics. These additional attributes permit to decide the validation of data, which are passed to the segmentation algorithm. With the segmentation the different founded clusters are rearranged and validated.

The results of the developed processing algorithms are tested with experimental data, acquired with a sensor prototype, and then displayed and analyzed with a debugging interface. The user may change the key parameters, which define the algorithms, with commands available in the debugging interface, so their values can be determined observing the experimental results. In Figure 11 an example of the debugging

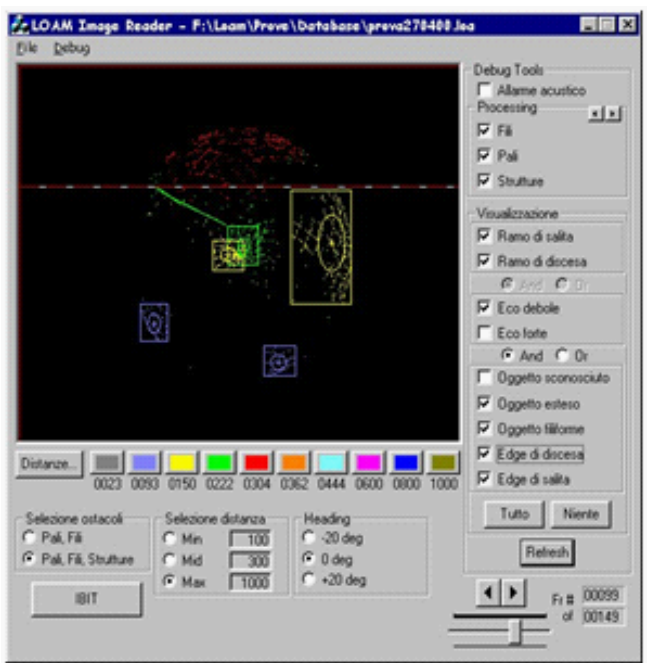

Figure 10: Debugging Interface with displayed results.

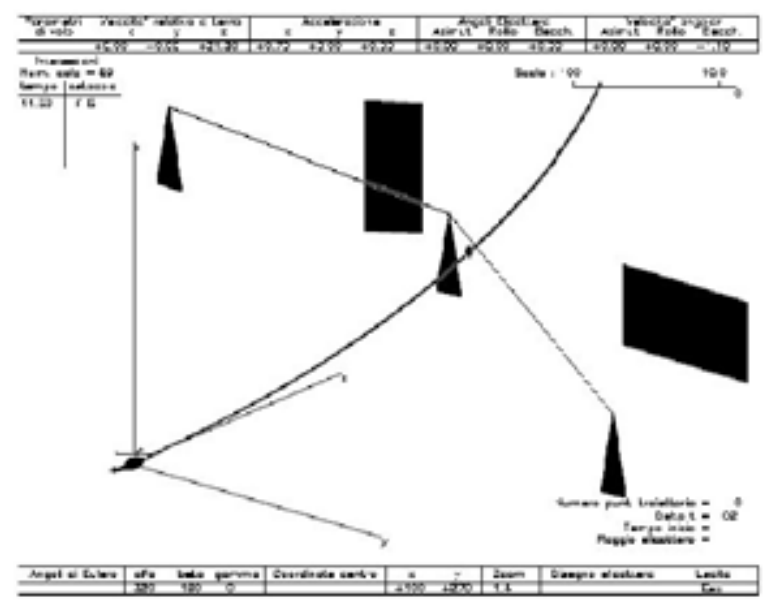

Figure 11: LTC Simulation Environment.

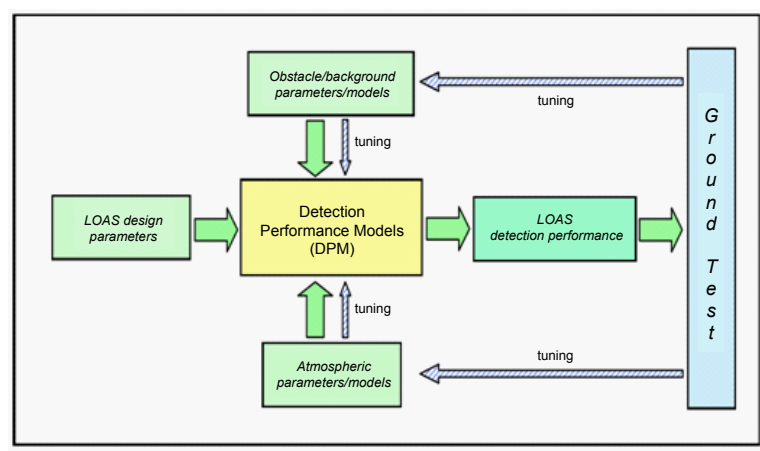

Figure 12: Detection performance models and ground test.

interface is shown. With the processing of currently available data, we see that the algorithms are capable to detect and classify the different obstacles, thanks to the flight testing currently performed, the parameters are being definitively set and optimized.

Processing of currently available data has shown that the algorithms now implemented are capable to detect and classify the different obstacles of interest, and flight-testing being carried out on helicopters and UAVs is giving the opportunity to definitively set and optimize the processing parameters.

\section{Calculation of Alternative Flight Paths}

In a laser obstacle detection and warning system, there is also the problem of providing only essential information to the pilot. LOAM scanner system, in fact, detects the position of every potential obstacle in the environment where the platform is moving. In a generic scenario, with many obstacles in the field of view of the warning system, it may be difficult for the pilot to monitor all of them. For this reason, a system able to discriminate the more dangerous obstacles and to supply the relative information is required.

To solve this problem, three algorithms have been implemented: calculation of next trajectory segment, calculation of possible intersections with obstacles and determination of an alternative (optimal) trajectory. A simulation environment was required to test and refine the algorithms performance. Therefore, to simulate the platform flight in a generic scenario, a three-dimensional environment 
Figure 12 has been implemented that allows seeing, from different point of view, the scenario scanned from laser sensor. In addition, this environment permits to observe the trajectory's trace followed by the platformr (trajectory from proper algorithm calculated) and verify the intersection with the obstacles. The simulation allows to modify smoothly the flight parameters and to observe the platform motion and the forces acting on it.

The main functions performed by the algorithms are essentially two: calculation of the next trajectory and determination of possible intersections between the platform and the obstacles located in the scenario and detected by laser scanner.

In the case of manned and unmanned rotocraft, the calculation problem for the trajectory is a more difficult task due to the complexity of this type of aircraft and implies a careful study of his flight dynamic. The trajectory followed by the platform and the supposed flight path in the subsequent twenty seconds, is extrapolated from motion's data supplied by navigation's system: the velocity relative to earth, the velocity relative to air, the acceleration and the angles describing the platform orientation.

The second algorithm is developed to search a possible intersection between the trajectory previously calculated and the obstacles acquired by laser scanner. Through the analysis of every single intersection, the algorithm provides a priority list of the most dangerous obstacles located in front of the aircraft. The algorithm is optimized to satisfy the performance, about precision and speed of execution, required.

\section{LOAM History Function}

Due to the restricted system field of view, some information, acquired in the previous frame, may be lost successively. To keep obstacles information when they are outside the present frame, it is necessary to store the position of every object detected and then update the coordinates with respect to the platform body-fixed reference system. LOAM history function stores the data of the obstacles for a defined time interval and deletes them when they are outside the platform possible trajectories (outside its flight envelope).

Since the motion data supplied from navigation system are, like every measure, affected by an error, we evaluate how these errors affect the positions calculated for every obstacle. To do so, a Gaussian error is added to every data and a statistic of the position error is calculated for obstacles near and far from the aircraft. When the impact warning processing establishes that the trajectory currently flown by the aircraft has a collision risk, the algorithm searches the corrections necessary to avoid the obstacles, and provides the pilot an indication about the alternative (optimal) direction to fly. The optimal trajectory is the one having the smaller possible correction (necessary to avoid the obstacles) and which is compatible with a safe flight path.

\section{LOAM Performance Prediction and Ground Test}

Ground trials of the LOAM system were performed in order to verify the system detection performance in various weather conditions, and to test the validity of the mathematical models used for performance calculations. This was particularly important for preparing the LOAM flight test activity. It was in fact necessary to define a criteria for determining the system detection range performances in the worst environmental conditions, and with the worst obstacle scenarios (i.e., small wires with low reflectivity), even without performing real tests in these conditions (i.e., using experimental data collected in fear weather and with average obstacles). Mathematical modeling and ground testing of the LOAM detection performance were therefore required in order to give proper weights to the parameters playing a role in realistic operational scenarios, and to determine the target LOAM detection performances to be demonstrated in flight. Figure 13 illustrates the process involved.

As the ground test activities permitted to validate the models developed, it was then possible to identify reference sets of obstacle, background and atmospheric parameters giving the absolute minimum performance of the LOAM system. This is illustrated in Figure 14. Obviously, the successive flight test activities were performed only in a small portion of the LOAM operational envelopes, but the results obtained could be extended to the entire envelopes by using the validated mathematical models.

For initial design calculations, the wire obstacle detection capability of the LOAM is modelled by the following simplified Signal to Noise Ratio (SNR) equation:

$S N R=\frac{4 E_{p} A_{r} L_{T} L_{r} e^{-2 \gamma R} d_{W} \rho}{\pi P_{D} R^{2}(\alpha R+D) N E P}$

where:

$\mathrm{EP}=$ output laser pulse energy

$\mathrm{Ar}=$ receiver aperture

$\mathrm{LT}=$ transmission losses (including beam shaping)

$\mathrm{Lr}=$ reception losses (including optical filter)

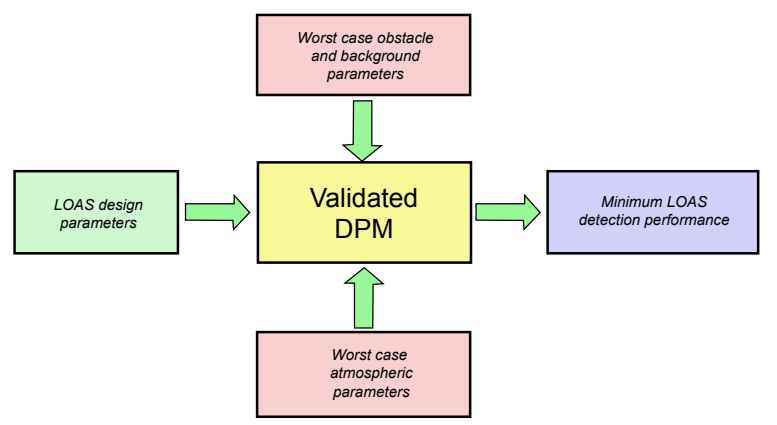

Figure 13: Minimum detection performance calculation.

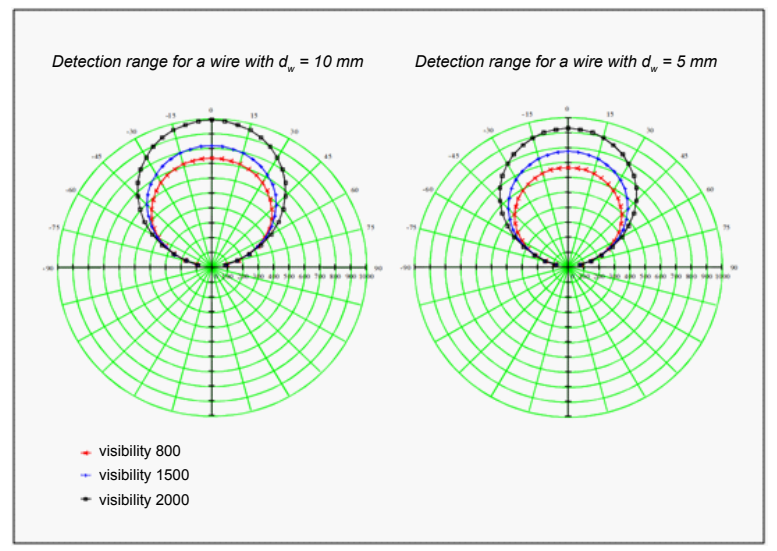

Figure 14: LOAM detection range performance for wires. 
$\gamma=$ atmospheric extinction coefficient $\mathrm{dW}=$ wire diameter

$\rho=$ wire reflectivity

$\mathrm{PD}=$ pulse duration

$\mathrm{R}=$ obstacle range

$\alpha=$ beam divergence $(1 / \mathrm{e} 2)$

$\mathrm{D}=$ initial beam diameter

\section{$\mathrm{NEP}=$ noise equivalent power}

The extinction coefficient $(\gamma)$ is calculated using the empirical model suggested by Elder and Strong [8] and modified by Langer [9]. Additionally, for propagation in rainy conditions, the equations developed by Middleton were adopted [10]. This approach (ESLM model) is particularly useful because it provides a means of relating the atmospheric transmission of the $i^{\text {th }}$ window to the atmospheric visibility, relative humidity and rainfall-rate (i.e., readily measurable parameters).

The first assumption made $[8,9]$ is that variations in the transmission are caused by changes in the water content of the air. Specifically, changes in the concentration of $\mathrm{H}_{2} \mathrm{O}$ cause changes in the absorption, and changes in the size and number of water droplets with humidity cause changes in the scattered component. This is a valid assumption since the other atmospheric constituents have a reasonably constant effect on the transmittance of a given atmospheric window.

It is customary to express the number of $\mathrm{H}_{2} \mathrm{O}$ molecules encountered by the beam of light in terms of the number of precipitable millimetres of water in the path. Specifically, the depth of the layer of water that would be formed if all the water molecules along the propagation path were condensed in a container having the same cross-sectional area as the beam is the amount of precipitable water. A cubic meter of air having an absolute humidity of $\rho$ grams per $\mathrm{m}^{3}$ would yield condensed water that cover an $1 \mathrm{~m}^{2}$ area and have a depth of:

$$
w^{\prime}=10^{-3} \rho
$$

where $w^{\prime}$ is the precipitable water having units of $\mathrm{mm}$ per meter of path length. For a path length of $z$ meters eq. (2) becomes:

$$
w=10^{-3} \rho \cdot z
$$

where $w$ is now the total precipitable water in millimetres. The value of $\rho$, the density of water vapour, can be obtained using the following equation [6], which is convenient for computer code implementation:

$$
\rho=1322.8 \cdot \frac{R H}{T} e^{\left[\frac{25.22 \cdot(T-273.16)}{T}-5.31 \cdot \ln \left(\frac{T}{273.16}\right)\right]}
$$

Where $R H$ is the relative humidity (as a fraction), and $T$ is the absolute temperature $\left({ }^{\circ} \mathrm{K}\right)$. Two empirical expressions, developed by Langer [9], can be used to calculate the absorptive transmittance $\tau_{a i}$ for the $i^{\text {th }}$ window for any given value of the precipitable water content. These expressions are:

$$
\begin{aligned}
& \tau_{a i}=e^{-A_{i} \sqrt{w}}, \text { for } w<w_{i} \\
& \tau_{a i}=k_{i}\left(\frac{w_{i}}{w}\right)^{\beta_{i}}, \text { for } w>w_{i}
\end{aligned}
$$

Where $A_{i}, k_{i}, \beta_{i}$ and $w_{i}$ are constants whose values for each atmospheric window are listed in $[8,9]$. For the LOAM laser wavelength $\left(\lambda=1550 \mathrm{~nm}-4^{\text {th }}\right.$ atmospheric window), $A_{i}=0.211, k_{i}=0.802, \beta_{i}=0.111$ and $w_{i}=1.1$.

In summary, eq. (5) and eq. (6), together with eq. (3) and eq. (4), provide information that can be used to obtain an estimate of the absorptive transmittance $\left(\tau_{a i}\right)$ of laser beams having wavelengths that fall within the various atmospheric windows. The results apply to horizontal paths in the atmosphere near sea-level and for varying relative humidity. To obtain the total atmospheric transmittance we must multiply $\tau_{a i}$ by $\tau_{s i}$ (i.e., the transmittance due to scattering only).

Based on rigorous mathematical approaches, the scattering properties of the atmosphere due to the aerosol particles are difficult to quantify, and it is difficult to obtain an analytic expression for the scattering coefficient that will yield accurate values over a wide variety of conditions. However, an empirical relationship that is often used to model the scattering coefficient [5] has the form:

$$
\beta(\lambda)=C_{1} \lambda^{-\delta}+C_{2} \lambda^{-4}
$$

Where $C_{1}, C_{2}$, and $\delta$ are constants determined by the aerosol concentration and size distribution, and $\lambda$ is the wavelength of the radiation. The second term accounts for Rayleigh scattering. Since for all wavelengths longer than about $0.3 \mu \mathrm{m}$ the second term is considerably less than the first, it may be neglected. It has been found that produces reasonable results when applied to aerosols with a range of particle sizes. An attempt has also been made to relate $\delta$ and $C_{1}$ to the meteorological range. The apparent contrast $C_{z}$, of a source when viewed at $\lambda=0.55 \mu \mathrm{m}$ from a distance $z$ is by definition:

$$
C_{z}=\frac{R_{s z}-R_{b z}}{R_{b z}}
$$

where $R_{s z}$ and $R_{b z}$ are the apparent radiances of the source and its background as seen from a distance $z$. For $\lambda=0.55 \mu \mathrm{m}$, the distance at which the ratio:

$$
V=\frac{C_{z}}{C_{0}}=\frac{\frac{R_{s z}-R_{b z}}{R_{b z}}}{\frac{R_{s 0}-R_{b 0}}{R_{b 0}}}=0.02
$$

is defined as the meteorological range $V$ (or visual range). It must be observed that this quantity is different from the standard observer visibility $\left(V_{o b s}\right)$. Observer visibility is the greatest distance at which it is just possible to see and identify a target with the unaided eye. In daytime, the object used for $V_{\text {obs }}$ measurements is dark against the horizon sky (e.g., high contrast target), while during night time the target is a moderately intense light source. The International Visibility Code (IVC) is given in Table 2.

\begin{tabular}{|c|c|}
\hline Designation & Visibility \\
\hline Dense Fog & $0-50 \mathrm{~m}$ \\
\hline Thick Fog & $50-200 \mathrm{~m}$ \\
\hline Moderate Fog & $200-500 \mathrm{~m}$ \\
\hline Light Fog & $500-1 \mathrm{~km}$ \\
\hline Thin Fog & $1-2 \mathrm{~km}$ \\
\hline Haze & $2-4 \mathrm{~km}$ \\
\hline Light Haze & $4-10 \mathrm{~km}$ \\
\hline Clear & $10-20 \mathrm{~km}$ \\
\hline Very Clear & $20-50 \mathrm{~km}$ \\
\hline Exceptionally Clear & $>50 \mathrm{~km}$ \\
\hline Table 2: International Visibility Code (IVC).
\end{tabular}


It is evident that, while the range of values for each category is appropriate for general purposes, it is too broad for scientific applications. Visibility is a subjective measurement estimated by a trained observer and as such can have large variability associated with the reported value. Variations are created by observers having different threshold contrasts looking at nonideal targets. Obviously, visibility depends on the aerosol distribution and it is very sensitive to the local meteorological conditions. It is also dependent upon the view angle with respect to the sun. As the sun angle approaches the view angle, forward scattering into the line-of-sight increases and the visibility decreases. Therefore, reports from local weather stations may or may not represent the actual conditions at which the experiment is taking place. Since meteorogical range is defined quantitatively using the apparent contrast of a source (or the apparent radiances of the source and its background) as seen from a certain distance, it eliminates the subjective nature of the observer and the distinction between day and night. Unfortunately, carelessness has often resulted in using the term "visibility" when meteorological range is meant. To insure that there is no confusion, "observer-visibility" $\left(V_{o b s}\right)$ will be used in this thesis to indicate that it is an estimate.

If only $V_{o b s}$ is available, the meteorological range $(V)$ can be estimated [11] from:

$$
V \approx(1.3 \pm 0.3) \cdot V_{o b s}
$$

From eq. (10), if we assume that the source radiance is much greater than the background radiance (i.e., $R_{s}>R_{b}$ ) and that the background radiance is constant (i.e., $R_{b o}=R_{b z}$ ), then the transmittance at $\lambda=0.55 \mu \mathrm{m}$ (where absorption is negligible) is given by:

$$
\frac{R_{s v}}{R_{s 0}}=e^{-\beta V}=0.02
$$

Hence, we have:

$$
\ln \left(\frac{R_{S V}}{R_{S 0}}\right)=-\beta V=-3.91
$$

and also:

$$
\beta=\frac{3.91}{V}=C_{1} \lambda^{-\delta}
$$

It follows from eq. (13) that the constant $C_{1}$ is given by:

$$
C_{1}=\frac{3.91}{V} \cdot 0.55^{\delta}
$$

With this result the transmittance at the centre of the $i^{\text {th }}$ window is:

$$
\tau_{s i}=e^{-\frac{3.91}{V} \cdot\left(\frac{\lambda_{i}}{0.55}\right)^{-\delta} \cdot z}
$$

where $\lambda_{i}$ must be expressed in microns. If, because of haze, the meteorological range is less than $6 \mathrm{~km}$, the exponent $\delta$ is related to the meteorological range by the following empirical formula:

$$
\delta=0.585 \sqrt[3]{V}
$$

where $V$ is in kilometres. When $V \geq 6 \mathrm{~km}$, the exponent $\delta$ can be calculated by:

$$
\delta=0.0057 \cdot V+1.025
$$

For exceptionally good visibility $\delta=1.6$, and for average visibility $\delta \approx 1.3$. In summary, eq. (17), together with the appropriate value for $\delta$, allows to compute the scattering transmittance at the centre of the $i^{\text {th }}$ window for any propagation path, if the meteorological range $V$ is known. It is important to note here that in general the transmittance will, of course, also be affected by atmospheric absorption, which depending on the relative humidity and temperature may be larger than $\tau_{s i}$

For LOAM, it is also very important to model propagation through haze and precipitation. Haze refers to the small particles suspended in the air. These particles consist of microscopic salt crystals, very fine dust, and combustion products. Their radii are less than $0.5 \mu \mathrm{m}$. During periods of high humidity, water molecules condense onto these particles, which then increase in size. It is essential that these condensation nuclei be available before condensation can take place. Since salt is quite hygroscopic, it is by far the most important condensation nucleus. Fog occurs when the condensation nuclei grow into water droplets or ice crystals with radii exceeding $0.5 \mu \mathrm{m}$. Clouds are formed in the same way; the only distinction between fog and clouds is that one touches the ground while the other does not. By convention fog limits the visibility to less than $1 \mathrm{~km}$, whereas in a mist the visibility is greater than $1 \mathrm{~km}$. We know that in the early stages of droplet growth the Mie attenuation factor $K$ depends strongly on the wavelength. When the drop has reached a radius $\mathrm{a} \approx 10 \lambda$ the value of $K$ approaches 2 , and the scattering is now independent of wavelength, i.e., it is non-selective. Since most of the fog droplets have radii ranging from 5 to $15 \mu \mathrm{m}$ they are comparable in size to the wavelength of infrared radiation. Consequently the value of the scattering cross section is near its maximum. It follows that the transmission of fogs in either the visible or $I R$ spectral region is poor for any reasonable path length. This of course also applies to clouds. Since haze particles are usually less than $0.5 \mu \mathrm{m}$, we note that for laser beams in the IR spectral region $a / \lambda<<1$ and the scattering is not an important attenuation mechanism. This explains why photographs of distant objects are sometimes made with infrared-sensitive film that responds to wavelengths out to about $0.85 \mu \mathrm{m}$. At this wavelength the transmittance of a light haze is about twice that at $0.5 \mu \mathrm{m}$. Raindrops are of course many times larger than the wavelengths of laser beams. As a result there is no wavelength-dependent scattering. The scattering coefficient does, however, depend strongly on the size of the drop.

Middleton [11] has shown that the scattering coefficient with rain is given by:

$$
\beta_{\text {rain }}=1.25 \cdot 10^{-6} \frac{\Delta x / \Delta t}{a^{3}}
$$

where $\Delta x / \Delta t$ is the rainfall rate in centimetres of depth per second and a is the radius of the drops in centimetres. Rainfall rates for four different rain conditions and the corresponding transmittance (due to scattering only) of a $1.8-\mathrm{km}$ path are shown in Table 3.

These data are useful for order of magnitude estimates. In order to obtain accurate estimates, the concentrations of the different types of rain drops (radius) and the associated rainfall rates should be known. In this case, the scattering coefficient can be calculated as the

\begin{tabular}{|c|c|}
\hline Rainfall (cm/h) & Transmittance (1.8 $\mathbf{~ k m ~ p a t h ) ~}$ \\
\hline 0.25 & 0.88 \\
\hline 1.25 & 0.74 \\
\hline 2.5 & 0.65 \\
\hline 10.0 & 0.38 \\
\hline
\end{tabular}

Table 3: Transmittance of a 1.8-km path through rain. 
Citation: Sabatini R, Richardson MA, Roviaro E (2013) Development and Flight Test of an Avionics Lidar for Helicopter and UAV Low-Level Flight. J Aeronaut Aerospace Eng 2: 114. doi:10.4172/2168-9792.1000114

Page 10 of 13

\begin{tabular}{|c|c|}
\hline Rain Intensity & Rainfall $(\mathbf{m m} /$ hour) \\
\hline Mist & 0.025 \\
\hline Drizzle & 0.25 \\
\hline Light & 1.0 \\
\hline Moderate & 4.0 \\
\hline Heavy & 16 \\
\hline Thundershower & 40 \\
\hline Cloud-burst & 100 \\
\hline
\end{tabular}

Table 4: Representative rainfall rates.

sum of the partial coefficients associated to the various rain drops. A simpler approach, used in LOWTRAN, gives good approximations of the results obtained with eq. (18) for most concentrations of different rain particles. Particularly, in LOWTRAN, the scattering coefficient with rain has been empirically related only to the rainfall rate $\Delta x / \Delta t$ (expressed in $\mathrm{mm} /$ hour), as follows [12]:

$$
\beta_{\text {rain }} \approx 0.365 \cdot\left(\frac{\Delta x}{\Delta t}\right)^{0.63}
$$

Table 4 provides representative rainfall rates which can be used in eq. (18) and (19), when no direct measurements are available, to obtain order of magnitude estimations of $\beta_{\text {rain }}$.

In the presence of rain, in addition to the scattering losses calculated with eq. (18) or (19), there are, of course, losses by absorption along the path, and these must be included in the calculation of the total atmospheric transmittance with rain.

In order to estimate the SNR from experimental LOAM detector current measurements (iSIG), obtained with certain obstacle ranges (R) and incidence angles $(\theta)$, SNR was expressed as follows:

$$
S N R=20 \log \left(\frac{i_{S I G}(R, \theta)}{i_{N O I S E}}\right)
$$

The noise current terms in eq. (20) was modeled as:

$$
i_{N O I S E}=\sqrt{i_{T H}^{2}+i_{B K}^{2}+i_{D K}^{2}+i_{R A}^{2}}
$$

where:

$i_{T H}=$ thermal noise current

$i_{B K}=$ background noise current

$i_{D K}=$ dark noise current

$i_{R A}=$ receiver amplifier noise

According to the LOAM design characteristics, we have:

$$
\begin{aligned}
& i_{B K}=\sqrt{2 q P_{S} P_{h} M_{A}\left(2+k M_{A}\right)} B \\
& i_{T H}=\sqrt{4 K_{B} \frac{T_{k} B k}{R_{L}}} \\
& i_{D K}=0.5 \cdot 10^{-12} \\
& i_{R A}=1.5 \cdot 10^{-12}
\end{aligned}
$$

where:

$$
\begin{aligned}
& P_{S}=\text { received solar power } \\
& P_{h}=\text { amplifier gain }
\end{aligned}
$$

$M_{A}=$ avalance multiplier

$k=$ noise factor of the avalance photodiode

$B=$ electronic bandwidth

$K_{B}=$ Boltzmann constant $\left(1.39 \times 10-23 \mathrm{~J} /{ }^{\circ} \mathrm{K}\right)$

$T_{k}=$ absolute temperature $\left({ }^{\circ} \mathrm{K}\right)$

$R_{L}=$ amplifier load resistance

The following characteristics were defined for a wire type obstacle according to LOAM operational requirements:

- Diameter: $5 \mathrm{~mm} \leq \mathrm{DW} \leq 70 \mathrm{~mm}$

- Shape: twisted or round

- Reflection: Purely diffuse (Lambertian)

- Reflectivity: $\geq 20 \%(\theta=0)$

The reference environmental parameters were set as follows:

- Visibility: $\quad \mathrm{V} \geq 800 \mathrm{~m}$

- Humidity: $\mathrm{RH} \leq 100 \%$

- Temperature: $\mathrm{T} \leq 50^{\circ} \mathrm{C}$

- Rain: Light/Medium/Heavy

- Background: $P_{B}=50 \mathrm{~W} / \mathrm{m}^{2} \mathrm{sr} \mu \mathrm{m}$

For calculation purposes, the $i_{S I G}(R, \theta)$ term in eq. (26), was modelled as:

$$
i_{S I G}=\sqrt{\frac{P_{T} d_{W} \rho D_{a}^{3} \eta e^{-2 \gamma R}}{4 R^{3} \lambda} \cdot \frac{P_{h}}{K_{a}} \cdot \frac{1}{R_{L}}}
$$

where:

$P_{T}=$ transmitted power

$P_{h}=$ amplifier gain

$D_{a}=$ aperture diameter

$K_{a}=$ aperture illumination constant $=\operatorname{sen}(\theta)^{5.4}$

Results of range performance calculations with various visibilities and with all other parameters set to the worst case are shown in Figure 15.

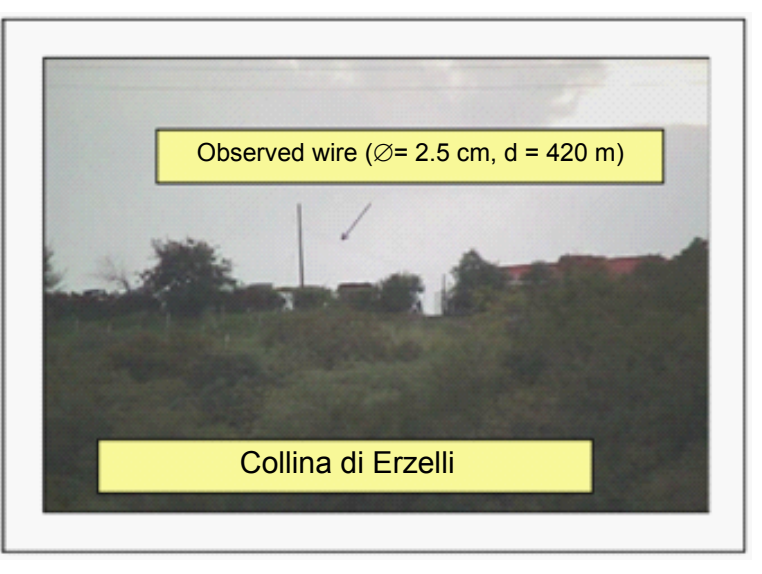

Figure 15: LOAM ground tests scenario. 
The false alarm probability is modelled as:

$$
P_{f a}=\frac{1}{B \cdot T_{f a} \cdot \eta}
$$

where:

\section{$B=$ receiver bandwidth}

$T_{f a}=$ mean time between false alarms

$\eta=$ maximum useful/non-ambiguous range

The mean time between false alarms corresponds to elementary electrical false alarms at the receiver level. The probability to have several false alarms on a straight line pattern is much lower. Statistically, these phenomena are described by the False Alarm Rate (FAR) and Detection Probability $\left(P_{d}\right)$. If the noise and signal distributions are known, the SNR can be estimated and the corresponding $D_{P}$ and FAR can be determined. According to the Rice calculation [11], the average FAR for the LOAM system is given by:

$$
\overline{F A R}=\frac{1}{2 \tau \sqrt{3}} \exp \left(-\frac{I_{t}^{2}}{2 I_{n}^{2}}\right)
$$

where:

$\tau=$ Electrical pulse length

$I_{t}=$ Threshold current

$I_{n}=$ Average noise current The LOAM $P_{d}$ is determined using pure Gaussian statistics $[13,14]$ :

$$
P_{d}=\frac{1}{\sqrt{\pi}} \int_{\frac{I_{t}-I_{n}}{\sqrt{2} I_{n}}}^{\infty} \exp \left(-\frac{i_{n}^{2}}{2 I_{n}^{2}}\right) d\left(\frac{i_{n}}{\sqrt{2} I_{n}}\right)
$$

$I_{n}=$ average signal current

$i_{n}=$ instantaneous noise current

The false alarm probability $\left(P_{f a}\right)$ is given by:

$$
P_{f a}=\tau \cdot \overline{F A R}
$$

and the cumulative detection probability $\left(P_{D}\right)$ is given by:

$$
P_{D}=1-\sum_{i=0}^{m} C_{M}^{i} P_{d}^{i}\left(1-P_{d}\right)^{M-i}
$$

where:

$M=$ number of possible detections

$m=$ minimum number of detections required

The scenario in which ground tests were performed is shown in Figure 16.

Tests were performed in various weather conditions (i.e., clear weather with $10 \leq V \leq 15 \mathrm{~km}$, and light/medium/heavy rain), using a wire of known section and reflectivity $\left(D_{W}=2.5 \mathrm{~cm}\right.$ and $\left.\rho=40 \%\right)$. The sets of data collected in clear and rainy weather conditions are shown in Figure 17. Form these data, it was evidenced that the returned signal power fluctuates independently from pulse to pulse according to a Gaussian distribution.

A comparison between the SNR predicted $\left(\mathrm{SNR}_{\mathrm{p}}\right)$ with $\gamma$ calculated using the ESLM model $\left(0.19 \mathrm{~km}^{-1} \leq \gamma \leq 0.22 \mathrm{~km}-1\right.$ for clear weather and $1.23 \mathrm{~km}^{-1} \leq \gamma \leq 2.94 \mathrm{~km}^{-1}$ for rainy conditions), assuming a

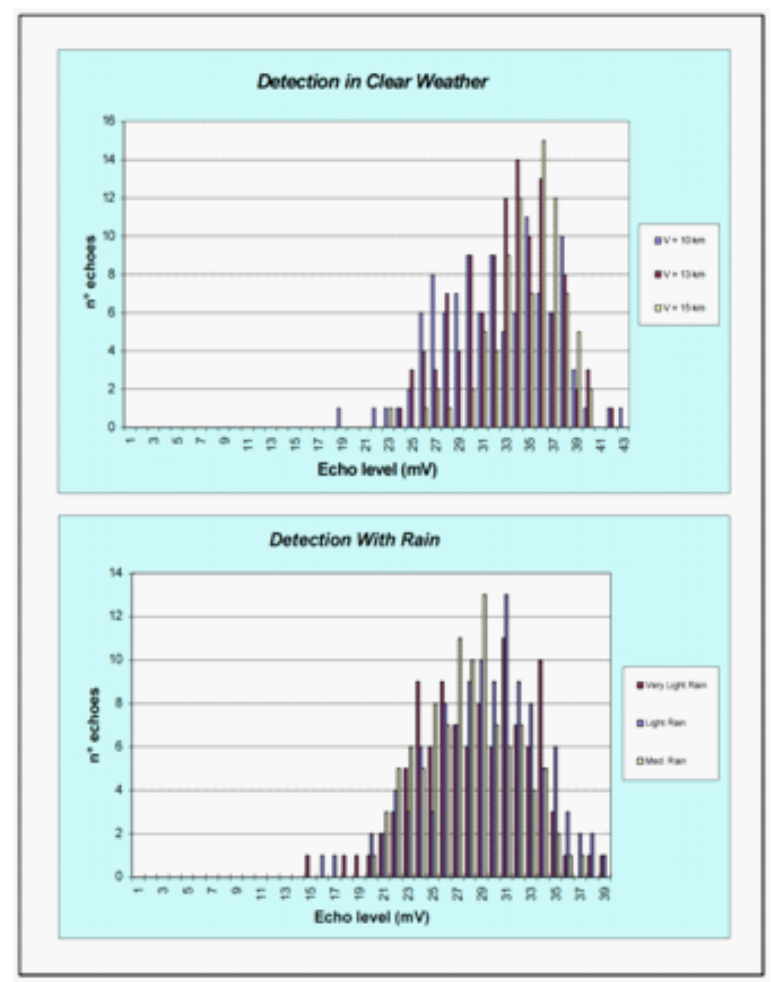

Figure 16: LOAM detection performance.

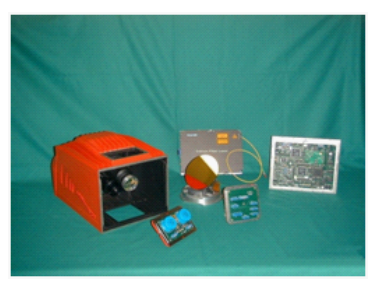

(a)

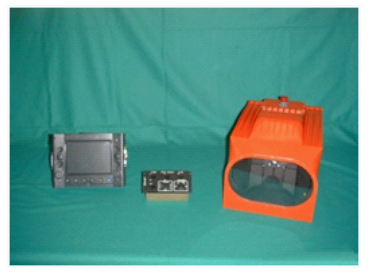

(b)
Figure 17: LOAM prototype used in the trials.

\begin{tabular}{|c|c|c|c|c|c|c|}
\hline & \multicolumn{3}{|c|}{ Clear Weather } & \multicolumn{3}{c|}{ Rain } \\
\hline & $\mathrm{V}=10 \mathrm{~km}$ & $\mathrm{~V}=12.5 \mathrm{~km}$ & $\mathrm{~V}=15 \mathrm{~km}$ & Light & Medium & Heavy \\
\hline $\mathrm{SNR}_{\mathrm{P}}$ & $4.90 \times 10^{4}$ & $4.95 \times 10^{4}$ & $5.02 \times 10^{4}$ & $3.14 \times 10^{4}$ & $1.83 \times 10^{4}$ & $1.45 \times 10^{4}$ \\
\hline $\mathrm{SNR}_{\mathrm{E}}$ & $3.35 \times 10^{4}$ & $3.80 \times 10^{4}$ & $4.27 \times 10^{4}$ & $2.87 \times 10^{4}$ & $2.47 \times 10^{4}$ & $2.13 \times 10^{4}$ \\
\hline
\end{tabular}

Table 5: LOAM predicted and measured SNR's.

background power of $10 \mathrm{Watt} / \mathrm{m}^{2} / \mathrm{sr} / \mu \mathrm{m}$ and $\rho=0.5$, and estimated from experimental data $\left(\mathrm{SNR}_{\mathrm{E}}\right)$, is shown in Table 5 .

\section{LOAM Flight Test Activities}

Figure 18 shows the LOAM prototype used for the flight trials. Particularly, the LOAM sub-units are shown in Figure 18a, while the pilot interface units are shown in Figure 18b.

Two different platforms were used for the tests. Figure 19 shows the LOAM installed on the NH-300 helicopter.

Figure 14 LOAM prototype units installed on NH-500.

Figure 20 shows the LOAM Sensor Head mounted on the second test platform (Agusta AB-212 helicopter). 


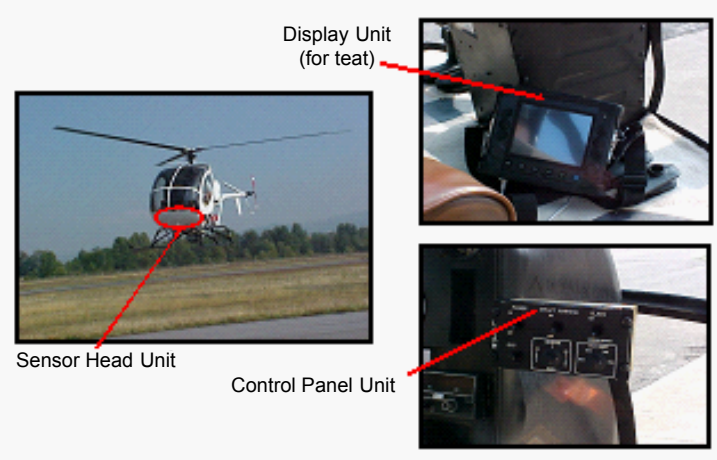

Figure 18: LOAM prototype units installed on $\mathrm{NH}-500$.

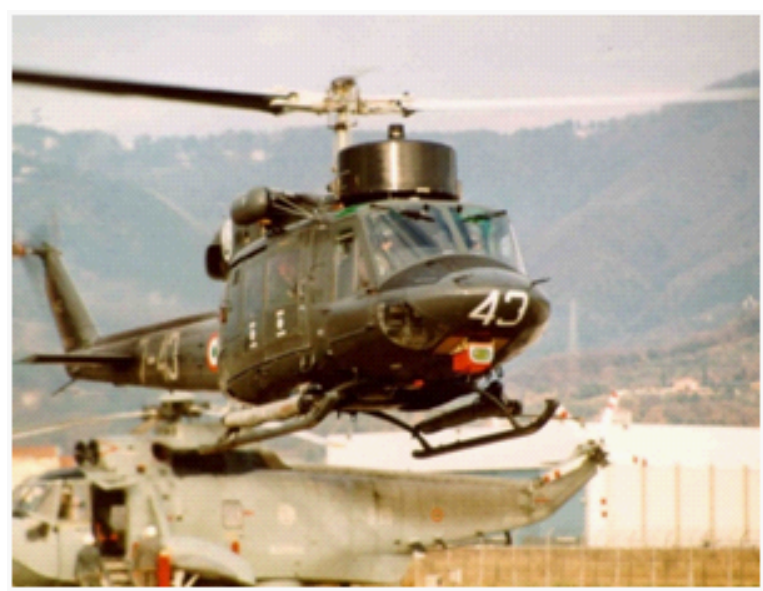

Figure 19: LOAM installed on AB-212.

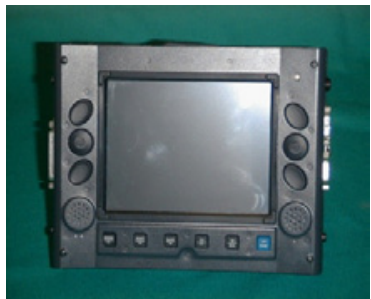

- $\mathbf{a}$ -

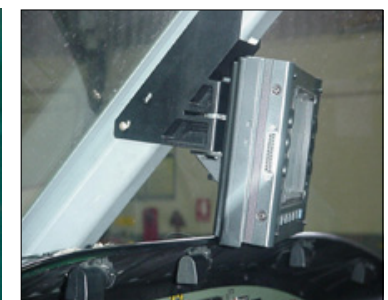

- b -
Figure 20: LOAM Display Unit on AB-212.

The Cockpit Display Unit (CDU) used for the trials is shown in Figure 21a. As shown in Figure 21b, the LOAM CDU was installed at the centre of the AB-212 glareshield, in order to be accessible to both pilot and co-pilot.

For the AB-212 test campaign, the LOAM Main Control Unit (MCU) was installed in the centre of the middle-console, as shown in Figure 22 (in a position accessible to both pilot and co-pilot). During the test flights, a Flight Test Engineer also operated a computer, linked to the LOAM system and displaying in real-time a 3-dimensional image reconstructed using the LOAM data. All images were recorded for the successive data analysis.

The results of this test campaign were very satisfactory. Particularly,

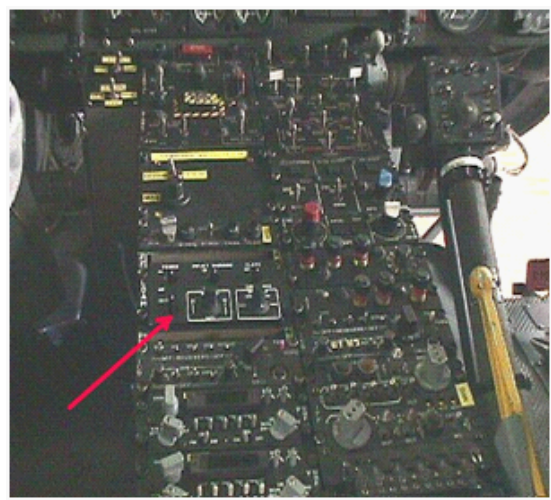

Figure 21: LOAM Main Control Unit on AB-212.

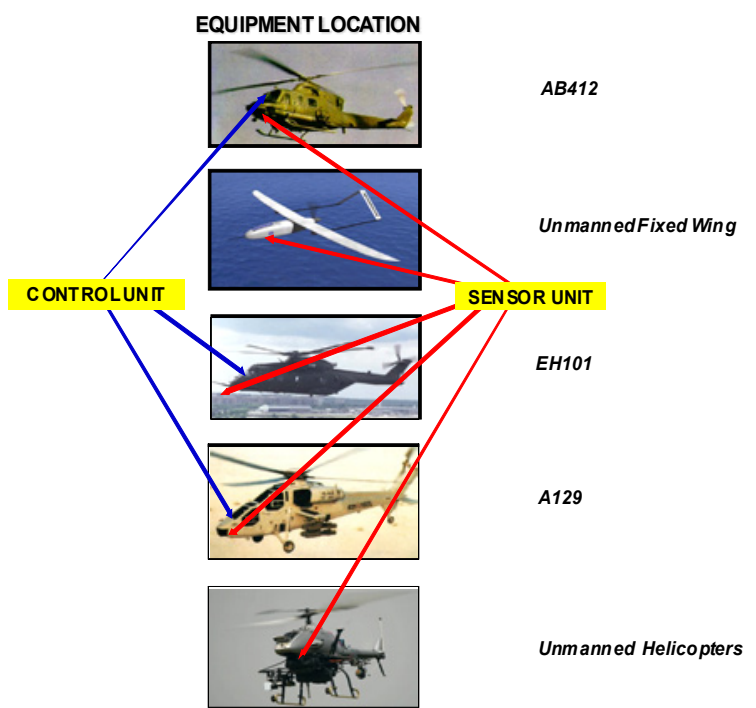

Figure 22: Candidate platforms for future LOAM flight tests.

the LOAM range performance were in accordance with the predictions and the LOAM detection/classification data processing algorithms were validated (detection and classification of all obstacles encountered was performed successfully). Furthermore, it was verified that the LOAM "History Function" was correctly implemented.

Future tests will be performed in order to finally assess the LOAM system performance (sensor and processing algorithms) in day/night with various weather/environmental conditions and to optimize the system Human Machine Interface both in helicopters and UAV (ground operator HMI). The candidate platforms for future LOAM integration and flight test activities are shown in Figure 18.

For future UAV platform flights, a dedicated control unit is being designed. Its characteristics are similar to the MCU developed for the initial helicopter flights. However, as this MCU has to be operated by the ground UAV pilot, in this case the LOAM operating modes are activated using two different communication data links for Line-ofSight (LOS) and Beyond LOS (BLOS) operations. Additionally, the LOAM display functions will be fully integrated in the UAV remote control position and the required LOAM display formats displayed to the UAV pilot in real-time [15-17]. 
Citation: Sabatini R, Richardson MA, Roviaro E (2013) Development and Flight Test of an Avionics Lidar for Helicopter and UAV Low-Level Flight. J Aeronaut Aerospace Eng 2: 114. doi:10.4172/2168-9792.1000114

Page 13 of 13

\section{Acknowledgements}

This activity was funded by the Italian Ministry of Defence $(M o D)$ under R\&D contract No. 2097-22-12-2000. The Italian Air Force Flight Test Centre developed, during a $\mathrm{PhD}$ research conducted at Cranfield University, the mathematical models, simulation tools and test facilities required for system ground and flight test. The authors wish to thank the personnel of SELEX-ELSAG, LOT-ORIEL and the Italian MoD Laser Test Range for helping in the preparation and execution of the ground and flight test activities.

\section{References}

1. Kellington CM (1975) An Optical Radar System for Obstacle Avoidance and Terrain Following.

2. Goldstein BS, Dalrymple GF (1967) Gallium arsenide injection laser radar Proceedings of the IEEE 55: 181-188.

3. The Anglo-French Compact Laser Radar demonstrator programme.

4. Buechtemann W, Eibert M (1995) Laser Based Obstacle Warning Sensors for Helicopters. AGARD.

5. Holder S, Branigan R (1994) Development and Flight Testing of an Obstacle Avoidance System for US Army Helicopters.

6. Selex SPA (2001) Technical Document TS021. LOAM System Characteristics.

7. Selex SPA (2001) Technical Document TS022. LOAM Sensor Head Unit Description.
8. Elder T, Strong J (1953) The infrared transmission of atmospheric windows. J Franklin I 255: 189-208.

9. Langer RM (1957) Signal Corps Report n DA-36-039-SC-72351.

10. Kneizys FX, Shuttle EP, Abreau LW, Chetwynd JH, Anderson GP, et al. (1988) Users Guide to LOWTRAN 7. Air Force Geophysical Laboratory Report AFGLTR-88-0177 Hansom AFB.

11. Middleton WEK (1952) Vision through the atmosphere. University of Toronto Press Canada.

12. Selex SPA (2001) Report V81, Programma di prove del sistema LOAM su elicottero AB212.

13. Skolnik M (2008) Radar Handbook. 3rd edn. McGraw-Hill Education, India.

14. Jelalian AV (1992) Laser Radar Systems. Artech House, United Kingdom.

15. Sabatini R (1998) Tactical Laser Systems Performance Analysis in Various Weather Conditions. RTO SET Symposium on "E-O Propagation, Signature and Syatem Performance Under Adverse Meterological Conditions Considering Out-of-Area Operations.

16. Thomas ME, Donald DD (1996) Atmospheric Transmission. ERIM-SPIE IR\&EO Systems.

17. Sabatini R, Richardson MA (2010) Airborne Laser Systems Testing and Analysis. NATO Research and Technology Organization Flight Test Techniques Series 26 .
Citation: Sabatini R, Richardson MA, Roviaro E (2013) Development and Flight Test of an Avionics Lidar for Helicopter and UAV Low-Level Flight. J Aeronaut Aerospace Eng 2: 114. doi:10.4172/2168-9792.1000114
Submit your next manuscript and get advantages of OMICS Group submissions

Unique features:

- User friendly/feasible website-translation of your paper to 50 world's leading languages

- Audio Version of published paper

Digital articles to share and explore

Special features:

250 Open Access Journals

20,000 editorial team

21 days rapid review process

Quality and quick editorial, review and publication processing

Indexing at PubMed (partial), Scopus, EBSCO, Index Copernicus and Google Scholar etc

- Sharing Option: Social Networking Enabled

- Authors, Reviewers and Editors rewarded with online Scientific Credits

Better discount for your subsequent articles

Submit your manuscript at: www.omicsonline.org/submission 